\title{
ASA-FTL: An Adaptive Separation Aware Flash Translation Layer for Solid State Drives*
}

\author{
Wei Xie \\ Department of Computer \\ Science \\ Texas Tech University \\ Lubbock, TX, USA \\ wei.xie@ttu.edu
}

\author{
Yong Chen \\ Department of Computer \\ Science \\ Texas Tech University \\ Lubbock, TX, USA \\ yong.chen@ttu.edu
}

\author{
Philip C. Roth \\ Computer Science and \\ Mathematics Division \\ Oak Ridge National \\ Laboratory \\ Oak Ridge,USA \\ rothpc@ornl.gov
}

\begin{abstract}
The flash-memory based Solid State Drive (SSD) presents a promising storage solution for increasingly critical dataintensive applications due to its low latency (high throughput), high bandwidth, and low power consumption. Within an SSD, its Flash Translation Layer (FTL) is responsible for exposing the SSD's flash memory storage to the computer system as a simple block device. The FTL design is one of the dominant factors determining an SSD's lifespan and performance. To reduce the garbage collection overhead and deliver better performance, we propose a new, low-cost, adaptive separation-aware flash translation layer (ASA-FTL) that combines sampling, data clustering and selective caching of recency information to accurately identify and separate hot/cold data while incurring minimal overhead. We use sampling for light-weight identification of separation criteria, and our dedicated selective caching mechanism is designed to save the limited RAM resource in contemporary SSDs. Using simulations of ASA-FTL with both real-world and synthetic workloads, we have shown that our proposed approach reduces the garbage collection overhead by up to $28 \%$ and the overall response time by $15 \%$ compared to one of the most advanced existing FTLs. We find that the data clustering using a small sample size provides significant performance benefit while only incurring a very small computation and memory cost. In addition, our evalu-

\footnotetext{
*This manuscript has been authored by UT-Battelle, LLC under Contract No. DE-AC05-00OR22725 with the U.S. Department of Energy. The United States Government retains and the publisher, by accepting the article for publication, acknowledges that the United States Government retains a non-exclusive, paid-up, irrevocable, world-wide license to publish or reproduce the published form of this manuscript, or allow others to do so, for United States Government purposes. The Department of Energy will provide public access to these results of federally sponsored research in accordance with the DOE Public Access Plan (http://energy.gov/downloads/doe-public-access-plan).
}

ation shows that ASA-FTL is able to adapt to the changes in the access pattern of workloads, which is a major advantage comparing to existing fixed data separation methods.

\section{INTRODUCTION}

Recently, there is a substantially growing demand on dataintensive computing in various fields, including many enterprise high end computing and scientific high performance computing applications. There is also a critical and enlarging gap between the application's I/O demand and the computing system's I/O capability. Data-intensive applications manipulate increasingly huge datasets and face challenges due to the unmatched storage system performance.

A high performance storage system is required for achieving highly efficient data processing and massive throughput for data-intensive problems. The NAND Flash based Solid State Drives (SSDs) are well suited for data-intensive applications due to their superior performance, low latency, and high bandwidth. They offer orders of magnitude higher throughput and less latency than the traditional hard disk drives. In addition, the feature of low energy consumption makes the SSDs even more attractive. Furthermore, many scientific and data-intensive applications feature random data access patterns that are not well served by traditional large-scale storage systems with spinning hard disk drives due to their relatively high access latency. In response, researchers have shown that storage systems using a massive number of SSDs 1] can give I/O performance benefits allowing computational chemistry and life sciences applications to compute problems at scales that were simply not feasible when using traditional storage systems 2]. A hybrid design combining SSDs and HDDs provides a costeffective way of improving the performance of the storage system 3 . 5. It has already been adopted in major IT companies and high performance computing facilities.

NAND flash memory is the core technology used in most SSDs due to their good balance between performance, capacity and cost. A typical SSD is usually composed of multiple NAND flash memory chips, a flash translation layer (FTL), a DRAM cache and some other necessary components. The smallest addressable unit of NAND flash memory is called a page, which ranges from $2 \mathrm{~KB}$ to $16 \mathrm{~KB}$ in size. Unlike other forms of memory, NAND flash memory pages must be erased before new data can be written to them. Unfortunately, the 
technology does not allow pages to be erased individually. Data are erased in units of erase blocks that are typically 64 to 128 pages in size. Because of the differing granularity for writes (page) and erasure (block), an in-place approach for writing data is very costly when using flash memory. Instead, these devices use an out-of-place 6] write approach whereby each write operation is appended to a log, similar to the approach used in a log-structured file system 7 .

On most SSDs, an FTL is responsible for maintaining the write log including management of free space and implementing the SSD's logical to physical address mapping. The design of this FTL has significant impact on the SSD's lifespan and performance. As with a traditional log-structured file system, the use of a write log requires more storage capacity compared to a non-log-based storage system. It also requires a costly garbage collection phase in case there are no free blocks available to satisfy incoming write requests. Random write workloads exacerbate the cost of this garbage collection due to fragmentation. In fact, fragmentation can cause SSD performance to degrade by an order of magnitude 8 9. Therefore, it is critical for an FTL to control the overhead and frequency of garbage collection.

Hot/cold data separation has shown to be beneficial for improving the FTL's performance and efficiency 6, 9,11 in terms of reducing the garbage collection overhead. In this study, we propose a new adaptive FTL hot/cold data separation method that meets the goal of controlling the cost of garbage collection. In this approach, data are classified as being either "hot" or "cold" indicating its likelihood of being overwritten in the near future. Hot data are likely to be overwritten, and thus are invalidated quickly, whereas cold data are less likely to be overwritten. By organizing hot data together, the FTL can reduce the need for copying data that would be invalidated anyway during garbage collection. Existing data separation approaches use predefined static thresholds and access frequency to classify whether data is hot or cold, and lack the ability to track the data hotness in SSDs online. In contrast, our proposed approach uses the last write access time distance as the criteria for identifying hot data, and clustering algorithm to adapt the FTL's behavior to the SSD's actual workload. To reduce the pressure on the device's limited on-device memory, we selectively cache the information about last write access time distance (i.e. inter-reference recency, IRR), similar to the caching approach used in demand-based selective caching of page-level address mapping (DFTL) 12. We use a natural clustering algorithm on sampled IRR 13. information for adaptive classification of hot/cold data. The clustering occurs periodically to update the separation critieria to adapt to any changes in the workload access pattern.

The main contributions of this work are:

- An innovative FTL approach for identifying and separating hot/cold data at the page level based on data clustering;

- A discussion of the use of sampling and selective caching to control the overhead of our clustering-based hot/cold data separation approach;

- A detailed description of the Hotness Updating Algo- rithm that samples and clusters data periodically to update the separation criteria and the overhead analysis of the approach;

- An evaluation of the proposed approach using the FlashSim simulator with several real world workloads, showing the performance benefits of our approach compared to a current state of the art FTL;

- An evaluation of the effect of the sample size and the adaptivity of the proposed ASA-FTL.

\section{BACKGROUND}

To better describe how our proposed approach works, we provide a brief overview of several related topics in this section.

\subsection{FTL Mapping Approaches}

SSD appears as a block device to the host system's operating system. Because of the FTL's log-based writing approach, the physical address of a data page may change several times during its lifetime. The FTL maintains mapping information between each page's logical address and its physical address. For good performance, the FTL's mechanism for maintaining this mapping should be very inexpensive. An FTL can be categorized based on the granularity of the information it keeps in its mapping table. A block-mapping approach has the smallest memory requirements, but its performance degrades rapidly when servicing small random writes. A page-mapping approach avoids this performance problem by storing all mappings at page-granularity, but it requires much more memory to store the mapping table, usually more than what is available in the device's on-board DRAM.

The FTL mapping approach is one important factor determining the FTL's garbage collection performance. If a block contains $N_{p}$ pages, the total free pages generated by erasing a victim block is $N_{p}$. If that victim block contained $N_{v}$ valid pages, the net gain is $N_{p}-N_{v}$, and the garbage collection efficiency is defined as $\alpha=\left(N_{p}-N v\right) / N_{p}$. Write amplification occurs due to the need to conduct extra writes when migrating valid pages during garbage collection.

In practice, SSDs use either a hybrid granularity mapping 14 15] or on-demand loading of parts of a larger page-level mapping table into the device's DRAM. Maintaining mapping information at the page granularity enables higher garbage collection efficiency than block-level or hybrid mapping approaches 12. The on-demand loading approach, also called dynamic FTL (DFTL) 12 is a state-of-the-art FTL approach that significantly outperforms hybrid granularity mapping schemes in terms of response time and garbage collection frequency and overhead, especially for workloads where random writes dominate.

\subsection{I/O Workload Access Pattern}

In real I/O workloads, accesses are rarely distributed uniformly in space and time. Instead, they exhibit both temporal and spatial locality 9]. In other terms, the I/O workload contains both hot and cold data; some pages are more frequently written/rewritten (hot data) and others are accessed in more of a write-once, read-many pattern (cold 


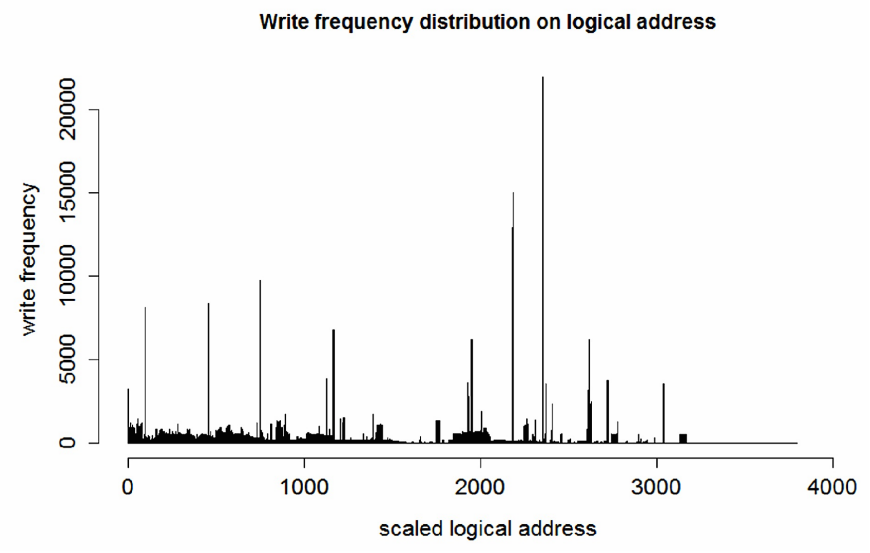

Figure 1: Locality and data hotness in the Financial1 workload. Frequency of writes to individual addresses (addresses are scaled from 0-4095).

data). With respect to the FTL, this non-uniformity is harmful because it can lead to low garbage collection efficiency, especially with block-level and hybrid FTL mapping approaches. Since write operations have the most direct impact on garbage collection overhead, read operations are usually not considered when examining the impact of workload locality on garbage collection.

Figure 1 illustrates the locality in write accesses in the Financial1 workload [16], showing that a small number of addresses are accessed much more frequently than the rest. By looking deeper into the access pattern over time, we find that there is little variation of the pattern, i.e. the access hotness of data, over a period of time. Figure 2 shows that the access pattern remains the same when comparing the first 5,000 and 50,000 write requests. This indicates that it is possible to accurately predict the access frequency of data using the historical hotness information. It might be not always true but we find that it applies to the traces we use in Section 4

\subsection{K-Means Clustering Algorithm}

The K-means algorithm 17] is one of the most widely used clustering algorithm. It paritions a group of numeric objects $\mathrm{X}$ into a pre-defined number of cluster that minimizes the sum of squared errors of the objects within each cluster. It requires initiating the $\mathrm{k}$ clusters' centroids so that the objects to be partioned are grouped into the clusters based on the Euclidean distance to the centroids. The assignment of the partitions to the objects are updated if a object is found to be closer to another cluster and it is done repeatedly until it is converged (a.k.a the sum of squares of each cluster does not change anymore).

The k-means algorithm is computationally expensive and requires time proportional to the product of the number of data items, number of clusters and the number of iterations. But the K-means algorithm is quite sensitive to initial positions of cluster centroids and the computation time can be largely reduced if the intial centroids are appropriately selecetd. The naive approaches either select the initial values randomly from the data points, or choose the first $\mathrm{k}$ samples of the data points. To improve the calculation time, a modified k-means clustering algorithm 18 has been proposed to better select the intial centroids and asigning the objects to the clusters, and is able to achieve the time complexity of $\mathrm{O}(\mathrm{nk})$ instead of $\mathrm{O}(\mathrm{nkl})$, where $\mathrm{n}$ is the number of objects to be clustered and $l$ is the number of iterations. For clustering data points into a fixed number of partitions, this modified K-means clustering algorithm can be executed within $\mathrm{O}(\mathrm{n})$ and can be practically used.

\section{ASA-FTL DESIGN}

In this section, we introduce the proposed adaptive separationaware flash translation layer (ASA-FTL) that reduces the garbage collection overhead and delivers better performance for SSDs. It combines sampling, data clustering, and selective caching of recency information to accurately identify and separate hot/cold data while incurring minimal overhead. We present the design motivation first, and then introduce the ASA-FTL design in detail.

\subsection{Design Motivation}

\subsubsection{Identification and Separation of Hot/cold Data}

The ability to classify data accurately as hot or cold is a key component of approaches for reducing the overhead of FTL garbage collection. It can sometimes be useful to also track warm data whose access pattern is not quite hot but really isn't cold either. Mixing hot with cold data in the same block will not be efficient because cold pages will be copied repeatedly as the hot pages are invalidated. Segregating the hot data and cold data into separate blocks will avoid repeated copying of cold data. There are several approaches for determining the hotness of a data page. A simple way to determine the data hotness is by using access frequency. As shown in Figure 1, the hot/cold data are illustrated by the height of the bars. Using the write frequency to approximate data hotness can be effective, but it does not consider the temporal locality of data. A more popular way of approximating hotness is to use the write interval of a page. The write interval is the time that a page stays valid between two updates. These write frequency or write interval information of each page must be maintained as an access table and available for fast access, which brings a challenge to the limited computation and memory resource in a SSD device. Using a traditional clustering algorithm, like K-means algorithm, to partition data according to the write intervals of each page is too costly, due to the large number of pages in an SSD. In general, identifying the hot data using write frequency or write intervals and clustering requires prohibitive computation and memory usage. This requirement motivates us to find a way to make the data separation in SSD more memory efficient and requires less computation complexity.

\subsubsection{Selective Caching and Sampling}

The hot/cold data separation requires two components: tracking the hotness of data and maintaining the separation criteria. To track the hotness of data, the traditional way is to maintain a data access table in the RAM and records the write frequency or write interval of each page of data. However, the limited memory resource in SSDs make it impractical. It is also not possible to maintain the access table 


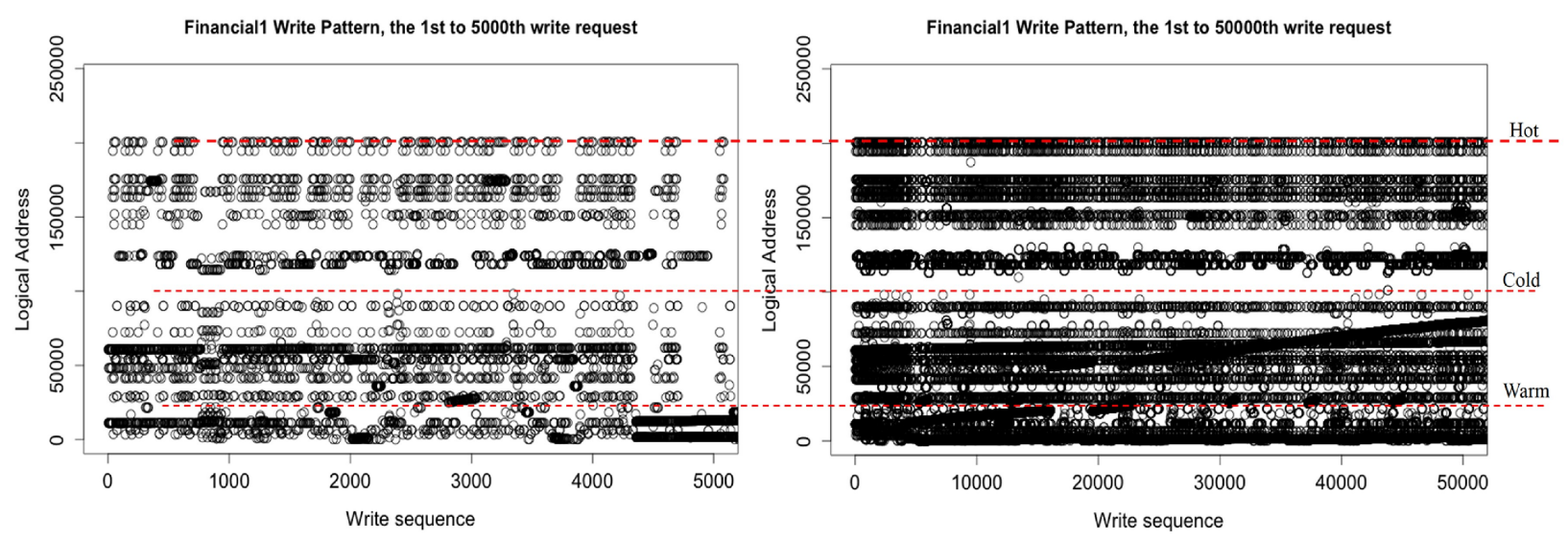

Figure 2: The write access pattern for the first 5,000 and 50,000 requests in Financial1 trace.

Table 1: Acronyms used in this paper.

\begin{tabular}{|l|l|l|l|}
\hline CMT & $\begin{array}{l}\text { Cache Mapping } \\
\text { Table }\end{array}$ & GTD & $\begin{array}{l}\text { Global Transla- } \\
\text { tion Directory }\end{array}$ \\
\hline CHT & $\begin{array}{l}\text { Cached Hotness } \\
\text { Table }\end{array}$ & ETB & $\begin{array}{l}\text { Extended Trans- } \\
\text { lation Block }\end{array}$ \\
\hline IRR & $\begin{array}{l}\text { Inter-reference } \\
\text { Recency }\end{array}$ & LWS & $\begin{array}{l}\text { Last Write Se- } \\
\text { quence }\end{array}$ \\
\hline SIT & $\begin{array}{l}\text { Sampled IRR Ta- } \\
\text { ble }\end{array}$ & & \\
\hline
\end{tabular}

solely in flash memory, because the access table will be frequently updated that flash memory is not fast enough. To solve this problem, we adopt a selective-caching mechanism similar to what is used in DFTL 12 , though we cache the hotness information of each page instead of the mapping information. The hotness information is cached in RAM and updated in-place when a cache-hit write occurs. When a page is replaced from the cache, an Age value is recorded with the page. The next time the page is written, the write interval is calculated and then loaded into the cache. In this way, the hotness information of the data pages is maintained and updated whenever written.

The clustering algorithm is generally time consuming if the data size is large. With a popular SSD configuration and with $128 \mathrm{~GB}$ capacity and $4 \mathrm{~KB}$ page size, there are more than 33 millions of data pages to be clustered. It would take tens of seconds for a dual-core desktop processor to cluster such a large data set, not mentioning the weak computation power of the embedded processor in SSDs. To minimize the computation overhead, we sample the hotness information of pages and use a small data size to feed the clustering algorithm in order to find the separation criteria. We further discuss the effect of the sample size in Section 4.5 with empirical results.

\subsection{Overall Design}

ASA-FTL maintains four major data structures (see Figure 3):

1. The Cached Mapping Table (CMT) that stores selected page mapping information, used to service incoming requests;

2. The Cached Hotness Table (CHT) that stores access recency and reuse distance information, used to determine data hotness;

3. The Global Translation Directory (GTD) that maps a logical address to the corresponding physical address of the translation block in flash memory or to the Extended Translation Block (ETB) structure used to service cache misses; and

4. The Sample Inter-reference Recency Table (SIT) that stores sampled inter-reference recency (IRR) [13. values for a randomly selected page, used to calculate the hot/cold data separation criteria.

For easy reference, the acronyms for these data structures, and other acronyms used to describe the ASA-FTL, are summarized in Table 1 It is noticed that since ASA-FTL is based on DFTL, some of the data structures, including the CMT and the GTD are inherited from the DFTL. But the CHT and SIT are added in order to track the hotness information of data in the SSD and the Translation Block is also extended to Extended Translation Block to persist the hotness information. The inter-reference recency (IRR) is also a technique borrowed from the LIRS algorithm [13] in order to track the recency of data access.

The GTD, SIT, CMT, and CHT data structures are maintained in the SSD's on-board RAM. Similar to DFTL, ASAFTL separates the total available flash memory blocks into data blocks and extended translation blocks (ETBs). The ETBs store mapping, and access (hotness) information for each page. To speed up address translation, this information is also selectively cached into the CMT and CHT. When a change of address mapping occurs (for example, when a page is written), the entry in ETB is loaded into the CMT and CHT, or updated if it was already there. The CMT entry will improve address translation time the next time the page is referenced. The CHT entry will improve the overhead of determining which Write Frontier (WF) to use the next time the page is written, as described later in this 


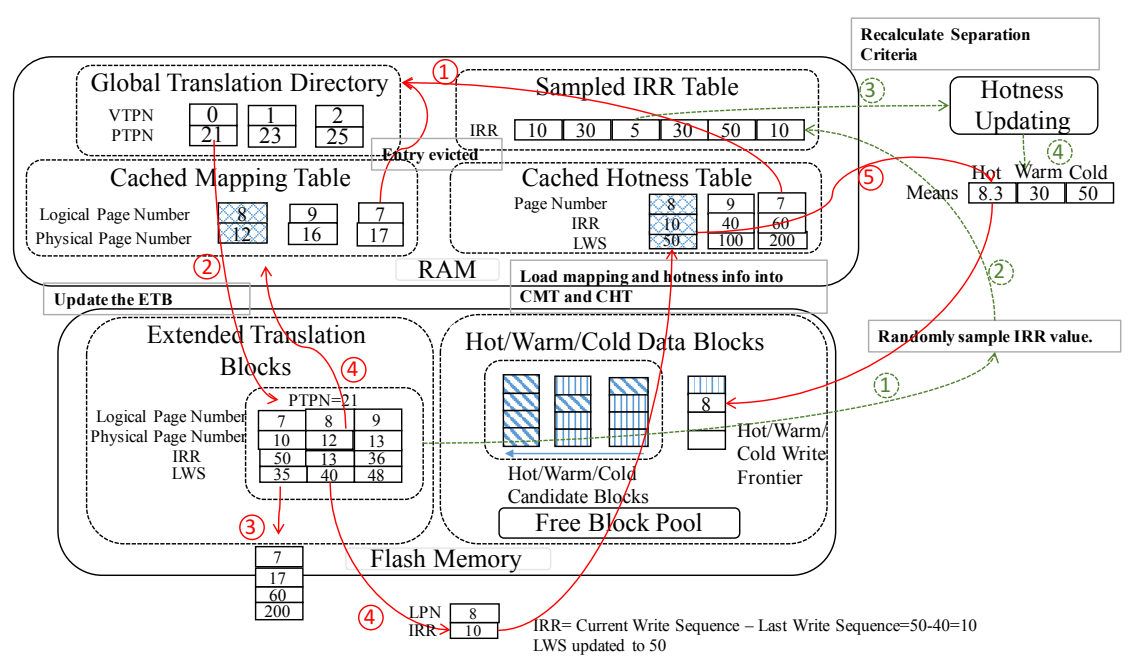

Figure 3: ASA-FTL architecture. Red, dashed arrows illustrate the steps taken when handling a write operation. Green, solid arrows illustrate steps taken when updating the clusters defining hotness criteria.

section. There is one WF each for hot data, warm data, and cold data.

The ETB access (hotness) information for a page includes its IRR and Last Write Sequence. In ASA-FTL, time is always represented as the write sequence, i.e. when a page is written to the SSD, the time advances by one. That being said, the the $I R R$ is the number of flash page writes between the second to last and last update of the page and it is used as the primary measure of the hotness of page; and the Last Write Sequence records the sequence when a page is lastly written. The Last Write Sequence is mainly used to calculate the IRR value when the page is written again. The details of how the IRR is calcualted at run-time will be discussed in Section 3.3

Each physical page in the SSD has the IRR and Last Write Sequence value alongside the Translation Block in the flash memory and can be loaded into the Cached Hotness Table in the RAM alongside the Cached Translation Table. A page's IRR is updated when a page is written then the Last Write Sequence is also updated-to-date.

ASA-FTL separates data blocks into three physical partitions corresponding to the data's hotness, similar to the multiple-pool approach [19]. Each partition has its own "Write Frontier" (WF), which is a block where the data belonging to the partition are written to. For example, a data page classified as "hot" is always written to the block that is allocated for accepting "hot" data. When a WF is fully written, it is added to the Candidate Block Pool (CBP) associated with that partition; then each of the hot, warm and cold CBP will only contain data that are classified as hot, warm and cold. The blocks in CBP are candidates for garbage collection the next time the garbage collector is invoked and the garbage collector is able to choose a hot, warm or cold data according to the specific GC algorithm. Multiple-pool approach is a core technique that enables hot/cold data separation, but as it is already widely used in existing studies, we do not further discuss the details of it.

It is important to determine which partition each data page should be placed on; this is because the effectiveness of hot/cold data separation highly depends on how accurate data are classfied according to the access skewness. To achieve natural and accurate separation, ASA-FTL clusters the IRR data using a widely-used K-means clustering algorithm with $K=3$, resulting in one cluster per partition. Because the K-means clustering algorithm is computationally expensive, we use an optimization of the K-means clustering algorithm that selects close-to-optimal intial centroids for each cluster so that the iterations of the algorithm can be much reduced. Again, because we only use a simple case of $\mathrm{K}$-means clustering ( $\mathrm{K}=3$ and one dimension data points), the computation cost is mostly determined by the number of data points to be clustered. In order to further reduce the cost, the IRR values of the pages are randomly sampled (simple random sampling method) and the sampled IRRs are used as the data points of the K-means clustering. The result of the K-means clustering serves as the centroids of the three partitions in ASA-FTL. When a page is written, ASA-FTL determines which partition should host the page by comparing the page's IRR against the centroids in each partition and is placed on the partition with the smallest distance.

The FTL runs a sampled clustering algorithm to re-calculate the separation criteria in order to keep up-to-date with the variance of the workload access pattern. Sampled clustering algorithm includes random sampling the IRR values, clustering the sampled IRR values to calculate the centroids for each cluster, and storing the centroids for each cluster to be used for separating written data into the three partitions. The solid lines ended with arrows in Figure 3 show 
an example of how ASA-FTL handles the writing of page 8 at time 50. In the example scenario, the $\mathrm{CMT} / \mathrm{CHT}$ are assumed to be full when the page is written, but page 8's information is not in the cache.

1. ASA-FTL selects page 7 as the victim page and uses the GTD to locate its entry within the ETB.

2. ASA-FTL uses the GTD to locate the mapping and hotness information for page 8 within the ETB.

3. ASA-FTL the CHT entry of page 7 is written back to the ETB of page 7 .

4. ASA-FTL calculates page 8's IRR by substracting the Last Write Sequence (LWS) (which is 40) from the current write sequence (which is 50), and then resets its LWS to the current write sequence (50).

5. ASA-FTL compares page 8's IRR against the mean IRR for each of the hotness partition clusters. At the time page 8 is written, those means are $8.3,30$, and 50 . Since page 8 's IRR of 10 is closest to 8.3 , page 8 is classified as hot and is assigned to the hot data partition.

\subsection{Online Data Hotness Tracking and Sepa- ration}

The ASA-FTL classifies and segregates a page based on its hotness value each time the page is written, regardless of whether the write was part of the SSD's workload or was caused by garbage collection. The first time a page is written, its LWS is set to the current write sequence and its IRR to a value representing infinity (all data are assumed cold initially).

When a page is written, ASA-FTL ensures the page's information is in the CMT and CHT. The CMT and CHT use a least recently used (LRU) replacement policy. When written, the entry for a page is moved to the head of a page list. If the page is written again before it is evicted, it is returned to the head of the list. If the CMT and CHT are full when a page is written, ASA-FTL selects a victim page from the tail of the list, and evicts this victim page to the ETBs by updating the victim page's eviction time, mapping information, and access information. It is noticed that the cache management part is the same as DFTL whereas the CHT is added in addition to the CMT.

Whenever a page is written and thus brought into the cache, its Last Write Sequence and IRR information are updated using equation 1, where the CWS is the current write sequence (a.k.a the total number of page writes since the SSD starts up).

$$
I R R=C W S-L W S
$$

The way that ASA-FTL categorizes a page's hotness is dynamically adaptive to the SSD's workload. The IRR value contains minimal historical information, and the IRR values of the SSD's collection of pages change as the workload pattern changes. The adaption to the change is handled by the sampled clustering algorithm which is further described in Section 3.4

\subsection{The Separation Criteria}

Even though the online tracking of the IRR values is able to capture variations of the access pattern, the criteria (a.k.a the centroids) that separates data into the three partitions also needs to be updated from time to time. The previously shown figure (see Figure 2 illustrates that the access pattern of a single application might be stable, but in a cluster that multiple applications utilize the storage at the same time, the access skewness can change significantly over time. In that case, the centroids calculated when a previous application was running may work sub-optimally for the current application. Given that, it is neccessary to keep the separation criteria up-to-date.

ASA-FTL's sampled clustering algorithm is responsible for maintaining the hotness clusters. Even though the random sampling and modified K-means clustering algorithm are used to reduce the cost of re-calculating the centroids for each of the hot/warm/cold partitions, it is still too much overhead if it is done frequently as it would incur a large number of reads from the SSD to gain the IRR values and utilization of the SSD's embedded CPU, which can be already busy to handle other tasks. The sampled clustering algorithm checks if there is enough variation in the data access pattern to initiate a re-calculation of the centroids for the partitions in order to reduce the occurence of the costly calculation. The variation of the data access pattern is measured by tracking the variation of the IRR values of each page. When a page is written, the existing IRR value is compared with the newly calcualted IRR value. Once the newly calculated IRR value classifies the page to a partition different from the existing IRR value, it is counted as an IRR miss. In addition, it tracks the number of data being written since the last time the clustering algorithm runs; then the IRR miss ratio can be defined as the ratio of writes that incur IRR misses. For every 1000 page writes (1000 is chosen because it is small enough to quickly adapt to the changes in the access pattern and a large enough sample size), the random sampling and the clustering are initiated when the IRR miss ratio exceeds a certain threshold. We tested different threshold values and found $50 \%$ is able to capture the variations we deliberately added to the workloads (by mixing different workloads).

When it is determined to re-calculate the centroids for each partitions, the sampled clustering algorithm starts to retrieve a basic random sample of the IRR values. The dotted lines ended with arrows shown in Figure 3 illustrate how the random sampling and clustering works.

1. The HUA samples as many $I R R$ values as possible into the SIT. (The size of the SIT determines the number of values sampled.)

2. The HUA uses a modified K-means clustering algorithm on the IRR values in the SIT to define the three hotness clusters.

3. The HUA computes the mean IRR for each of the clusters.

A modified K-means clustering algorithm that select the intial centroids with algorithm 18, is used to reduce the com- 
putational cost.

Because the number of pages stored in a typical SSD is so large, it is not feasible to cluster the $I R R$ values for all pages. There are too many $I R R$ values for the limited amount of SSD on-board RAM, and reading all $I R R$ values from the flash memory would be too time-consuming. Instead, ASAFTL randomly samples IRR values into the SIT stored in on-board RAM, and computes clusters using only those sampled $I R R$ values. If one byte is used to store a page's $I R R$ value (a reasonable assumption), a 16KB SIT supports sampling of 16,384 values. Because the $I R R$ values are sampled randomly from all pages, this number of samples will produce clusters that are a good representation of the entire collection of page $I R R$ values. The detailed evaluation of the effect of different sample sizes and the performance implication is further discussed in Section 4.5

\subsection{Overhead Analysis}

The storage and computation demands of the ASA-FTL are reasonable, given the capabilities of contemporary SSDs. The IRR and LWS for each page can each be stored in one byte. Consequently, for an SSD with 128GB flash memory, $512 \mathrm{MB}$ of flash capacity is needed to store the ETBs $(0.4 \%$ of the total storage capacity). The sizes of the CMT and CHT are determined by the size of the SSD's on-board RAM. Gupta et al showed that a CMT between $1 \mathrm{MB}$ and $4 \mathrm{MB}$ can provide close to $100 \%$ hit ratio for a variety of workloads 12]. ASA-FTL also requires space for its CHT, so approximately $2 \mathrm{MB}$ to $8 \mathrm{MB}$ should provide excellent ASAFTL hit ratios. With a smaller RAM size available for the cached tables, ASA-FTL should still provide performance improvement but would suffer from the cache replacement overhead.

The HUA introduces extra read operations to flash storage for sampling $I R R$ values from the ETB that are not present in other FTL approaches. But considering that HUA is usually not frequently triggered, this overhead does not incur a major penalty to the overall performance.

ASA-FTL's primary source of computation overhead is the $\mathrm{K}$-means clustering algorithm. The modified K-means algorithm has $O(n \log (n))$ time complexity, where $n$ is the number of values being clustered. When clustering 4096 IRR values, the computation overhead of the clustering algorithm is in the millisecond range. An approach that clusters the entire collection of $I R R$ values for a $2 \mathrm{~GB}$ SSD would require $1 \times 10^{8}$ times more computation time. Also, the HUA clusters $I R R$ values only when ASA-FTL detects that the workload pattern has changed. In all, the computation overhead of the ASA-FTL is kept at a reasonable level, especially considering the performance benefit ASA-FTL provides.

\section{EVALUATION}

To evaluate ASA-FTL, we implemented it in an SSD simulator and compared its behavior and performance with DFTL [12], a state-of-the-art FTL approach.

\subsection{Implementation}

To evaluate ASA-FTL, we implemented it in FlashSim 20], a widely-used SSD simulator. FlashSim is a trace-driven
Table 2: Parameters of the Simulated SSD Device

\begin{tabular}{|l|l|}
\hline SSD Capacity & $2 \mathrm{~GB}$ \\
\hline Block size/Page size & $128 \mathrm{~KB} / 2 \mathrm{~KB}$ \\
\hline Over-provisioning & $3 \%$ \\
\hline $\begin{array}{l}\text { Garbage collection } \\
\text { policy }\end{array}$ & Greedy \\
\hline Cache Size & $8 \mathrm{MB}$ \\
\hline
\end{tabular}

Table 3: Characteristics of Real World I/O Workloads

\begin{tabular}{|c|c|c|c|}
\hline $\mathrm{I} / \mathrm{O}$ traces & $\begin{array}{l}\text { Total } \\
\text { Write } \\
\text { Number } \\
\text { (GB) }\end{array}$ & $\begin{array}{l}\text { Total } \\
\text { Write } \\
\text { Number } \\
\text { (Count) }\end{array}$ & $\begin{array}{ll}\text { Average } & \\
\text { Write } & \text { Re- } \\
\text { quest } & \text { Size } \\
(\mathrm{KB}) & \end{array}$ \\
\hline Financial1 & 9 & 2097152 & 4.5 \\
\hline Cambridge & 46 & 1000000 & 48.8 \\
\hline TPCC1 & 4.6 & 484164 & 10.1 \\
\hline
\end{tabular}

simulator that adds a flash module to the DiskSim 21] simulator to model the behavior of flash memory-based SSDs. We implemented ASA-FTL into the FlashSim's FTL as an extension of DFTL, but it could also be integrated into most other FTL implementations.

The simulator schedules requests from an input I/O trace file into events that are handled by the simulator's flash module. When the simulator encounters a write request in the input trace, the request is intercepted by the ASA-FTL implementation and handled as described in Section 3

Table 2 summarizes the characteristics of the SSD we simulated for this study, and most were chosen because they are default parameters for FlashSim. We used a small capacity SSD (2GB) so as to more easily trigger garbage collection during the simulation. This helped to evaluate the write amplification effect described in Section 2, but does not diminish the generality of the experiment. We also used the Adaptive Replacement Cache (ARC) algorithm 22] for FlashSim's cache replacement.

\subsection{Workload Traces}

We used three real-world I/O workload traces as input for our simulations. The characteristics of these traces are summarized in Table 3 . The Financiall trace 16 was captured from a large financial institution's online transaction processing application, and is made available to the storage research community by the Storage Performance Council. The MSR Cambridge1 trace 23 was captured from an enterprise server at Microsoft Research Cambridge. The TPCC1 trace 23 was also collected by Microsoft, and reflects the workload experienced by a system when servicing the TPC$\mathrm{C}$ transaction processing benchmark.

\subsection{Performance Evaluation with Real World Workloads}

Figures 4 to 9 show simulation results using the real world workloads, for both ASA-FTL and the stock DFTL approaches. We use three metrics to compare the two approaches: the number of read and write operations during a garbage collection, the number of times a block was erased, 


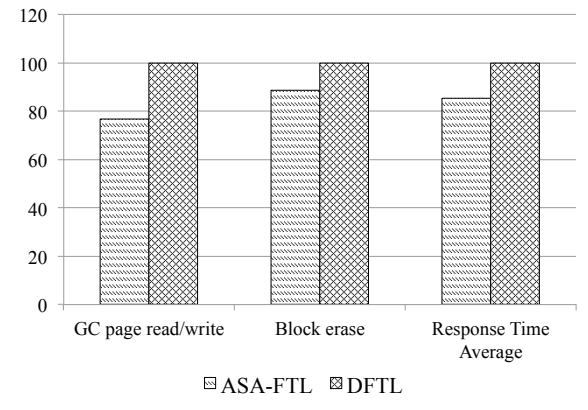

Figure 4: Normalized simulation results with Financial1 trace: ASA-FTL vs. DFTL.

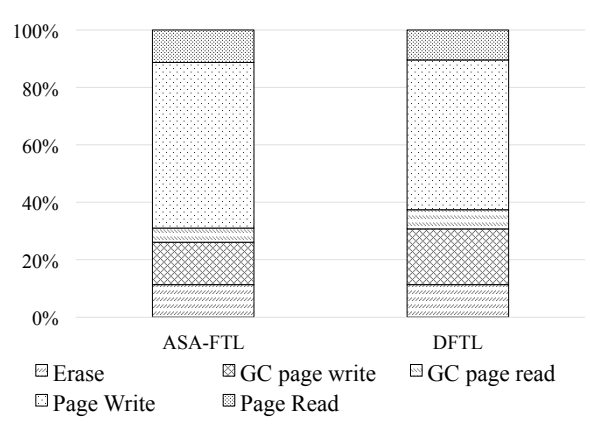

Figure 5: The proportion of the latency of each operation type with Financial1 trace: ASA-FTL vs. DFTL. The blocks with different pattern represent the proportion of the time spent in each of the operations as shown in the legend.

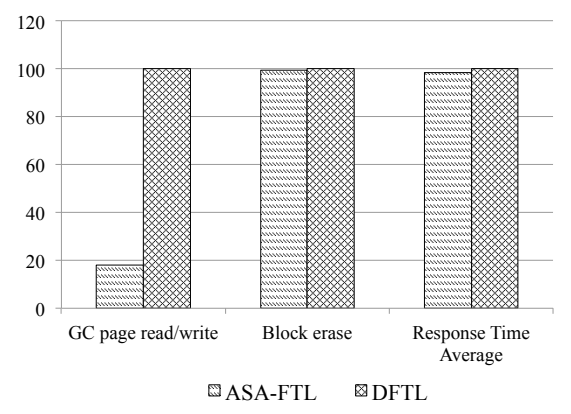

Figure 6: Normalized simulation results with MSR Cambridge trace: ASA-FTL vs. DFTL.

and the average response time. As shown in Figure4 for the Financial1 trace the number of read/write operations during ASA-FTL's garbage collection is approximately $24 \%$ less than for DFTL, and the erasure count approximately $12 \%$ less. Combined, these lead to a $15 \%$ improvement in average response time for ASA-FTL over DFTL. A large part of this overall improvement is due to ASA-FTL spending less time in writes during garbage collection (Figure 5). The reduction in erasure count is another significant factor contributing to ASA-FTL's performance advantage.

Figure 6 through Figure 9 compare our three evaluation metrics for ASA-FTL and DFTL for the Microsoft Cambridge trace and the TPCC1 trace. Interestingly, although ASAFTL performs five times fewer garbage collection read/write

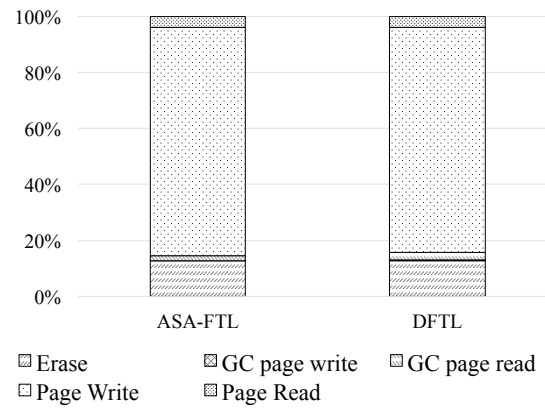

Figure 7: The proportion of the latency of each operation type with MSR Cambridge trace: ASA-FTL vs. DFTL. The blocks with different pattern represent the proportion of the time spent in each of the operations as shown in the legend.

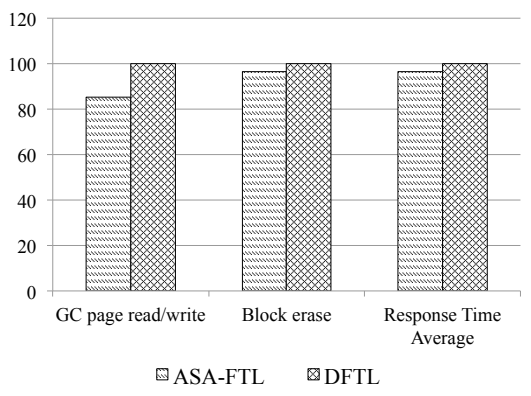

Figure 8: Normalized simulation results with TPCC1 trace: ASA-FTL vs. DFTL.

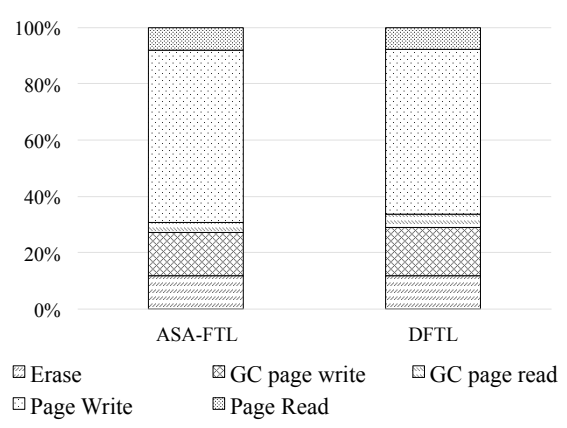

Figure 9: The proportion of the latency of each operation type with TPCC1 trace: ASA-FTL vs. DFTL. The blocks with different pattern represent the proportion of the time spent in each of the operations as shown in the legend.

operations than DFTL, the overall performance of the two approaches do not differ significantly. This is because the workload's requests are relatively large, and the garbage collection operations constitute a small part of each approach's overall response time. The garbage collection behavior with the MSR trace is more akin to that with the Financial1 trace, and ASA-FTL provides a non-negligible reduction in the number of garbage collection operations compared to DFTL. However, this reduction leads to a small improvement in overall response time, mainly because the benchmark's purpose results in a relatively uniform access distribution. 


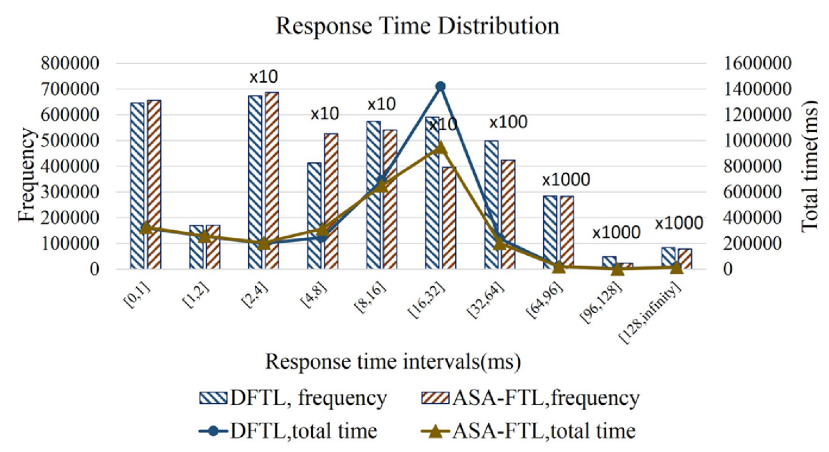

Figure 10: Distribution of response times when servicing the Financiall workload. For each time interval, the total time indicates the total time required to service all requests falling into that interval. For some of the time intervals, the frequency values are scaled by

Our evaluation using real-world workloads indicates that the behavior of ASA-FTL depends on the request size and locality in the workload. Flash-based SSDs work well for workloads with large request sizes. This is reflected in our results with the Cambridge trace: only about $5 \%$ of the total response time is attributed to read and copy operations during garbage collection, compared to about $20 \%$ for the Financial1 trace. Although garbage collection does not cause a significant overhead for workloads like Cambridge, ASA-FTL still helps reduce the number of times garbage collection is invoked and the need for copying pages, which translates to a decrease in the number of times a request is delayed if garbage collection is triggered.

Our results also show that the impact of the separation approach is dependent on the degree of access locality within the workload. Using the number of unique page addresses written during a sliding window of requests as a measure of locality, we expect that a large number of unique addresses written means the workload is more uniform, and a small number of unique addresses written means the workload is more skewed. Financiall exhibits substantially fewer unique pages accessed than TPCC1, which helps explain why the Financial1 workload benefits more from data separation. The Cambridge trace exhibits even greater locality: only 287 unique page addresses appear in the first 10000 write requests. This helps explain the dramatic decrease in garbage collection operations between ASA-FTL and DFTL. However, even with this highly skewed access distribution, the number of cold data pages might also be small, resulting in few occurrences of copying cold data and improving the number of garbage collection operations results in a minor benefit to overall performance from hot/cold data separation.

Based on our analysis, ASA-FTL delivers the most performance improvement for workloads that are dominated by small writes and exhibit a relatively high degree of locality. For such workloads, the moderate amount of cold data pages are separated from the hot data such that the cold data are less frequently found in the victim block selected by the garbage collector. ASA-FTL does not lead to significant performance improvement for other types of workloads, but does not significantly degrade performance either. For instance, garbage collection for workloads featuring large write requests tend to be efficient with existing strategies because the blocks involved tend to be written together (i.e., there is little fragmentation). For workloads that are relatively uniform, the simple greedy victim selection algorithm gives close-to-optimal performance 6]. For workloads whose write distribution is extremely skewed, the number of cold pages is small and copying of cold pages is not a significant contributor to overall performance. Although ASA-FTL does not provide a significant performance benefit for these types of workloads, but does not significantly degrade the performance either. Overall, ASA-FTL outperforms the state-ofthe-art DFTL approach.

Figure 10 shows a histogram of the observed response time for ASA-FTL and DFTL when servicing the Financiall workload. ASA-FTL showed significantly fewer instances of requiring between $8 \mathrm{~ms}$ and $64 \mathrm{~ms}$, with an especially large decrease in responses requiring $16 \mathrm{~ms}$ to $32 \mathrm{~ms}$. This reduction directly translates to the decrease in total response time of $32 \%$ in this interval. We believe the decrease in frequency and total time in the $16-32 \mathrm{~ms}$ interval is due mainly to reduced garbage collection overhead and frequency.

\subsection{Effect of Workload Skewness with Synthetic Benchmark}

To better understand the impact of workload distribution skew on ASA-FTL performance, we generated three synthetic I/O workload traces and used them as input to our ASA-FTL-enabled FlashSim simulator. In the Synth9/10 workload, $90 \%$ of incoming write requests go to $10 \%$ of the flash address space. In the Synth7/10 workload, $70 \%$ of write requests are for $30 \%$ of the space, and in the Synth $5 / 10$ workload, the incoming writes are uniformly distributed across the flash address space.

Figure 11 and Figure 12 show our evaluation metrics and a breakdown of the overall latency by type of flash operation for the three synthetic workloads. ASA-FTL exhibited a performance benefit of $13 \%, 3 \%$, and $0 \%$ for the Synth9/10, Synth7/10, and Synth5/10 workloads, respectively, as compared to DFTL. Thus ASA-FTL's performance benefit, which is mainly contributed by reduced garbage collection operations, is positively correlated with the amount of skew in the workload's write request distribution. Conversely, the general garbage collection efficiency of both ASAFTL and DFTL is negatively correlated with the workload skew; both are most efficient with the uniform request distribution of Synth5/10 (according to the fraction of GC related time in Figure 12. This suggests that higher skewness poses more problems in terms of GC overhead and thus more opportunity to be improved by ASA-FTL. Note that our evaluation results for Financial1 and Synth9/10 are qualitatively identical, as are those for TPCC1 and Synth7/10. This similarity suggests that TPCC1 has a similar distribution skew to Synth7/10, which is more uniform than that of Synth9/10 and Financial1. This observation reinforces our reasoning that ASA-FTL provided less benefit for TPCC1 than Financial1 because the TPCC1 workload had less skew in its request distribution. 


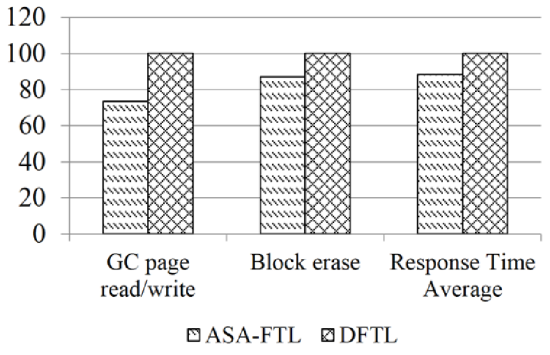

(a) Synth $9 / 10$

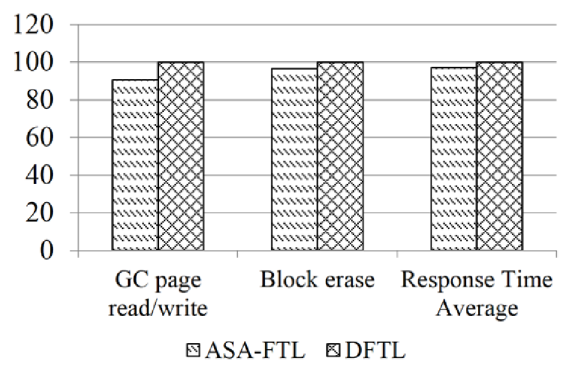

(b) Synth $7 / 10$

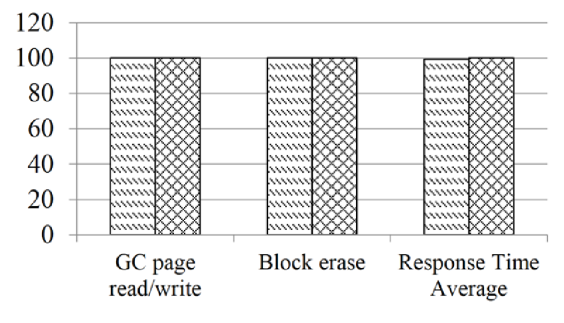

$\square A S A-F T L \otimes D F T L$

(c) Synth $5 / 10$

Figure 11: Normalized simulation results of synthetic workloads on ASA-FTL vs. DFTL

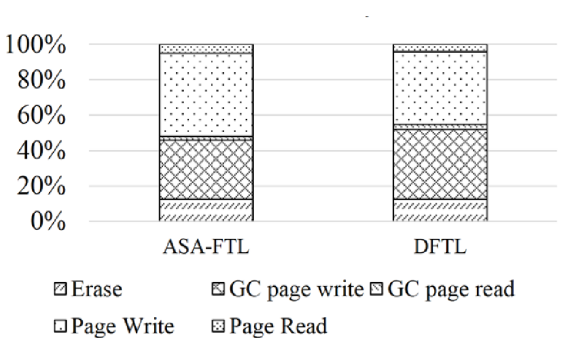

(a) Synth $9 / 10$

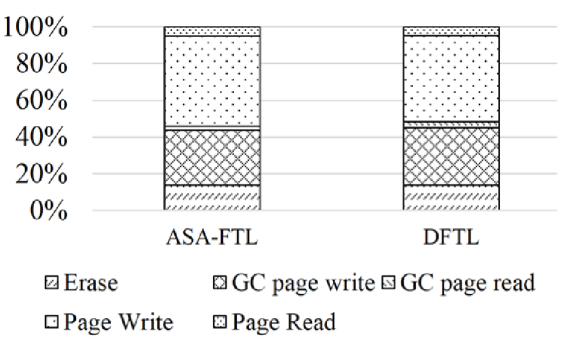

(b) Synth7/10

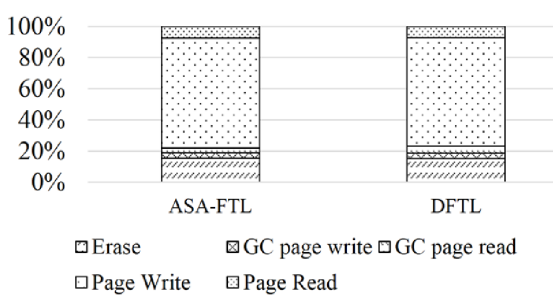

(c) Synth5/10

Figure 12: The proportion of the latency of each operation type: synthetic workloads with ASA-FTL vs. with DFTL. The blocks with different pattern represent the proportion of the time spent in each of the operations as shown in the legend.

\subsection{Effect of Sampling Size}

The sampling of the IRR values significantly reduces the RAM utilization and also the computation complexity of the data clustering. In this section, we evaluate the effect of the sampling size and how it affects the clustering computation overhead and performance. We use Financiall as the trace and sample different size of IRR data ranging from 1 million down to 10. The calculation time of the K-means clustering algorithm is recorded for these sample sizes. We also use the 1 million sample size as the baseline and evaluate the change of the average response time with smaller sample sizes. For each of the tests, we repeat the sampling and clustering process 10 times with different sampling seeds. As seen in Figure 13 as expected the calculation time of the clustering algorithm decreases abruptly, for example from 2.7 seconds to 0.19 seconds when the sample scales down from 1 million to 100 thousand. A sample size of 1000 only incurs $3.5 \%$ performance degradation in average and $2.8 \mathrm{~ms}$ calculation time, which are both acceptable. It is noticed that even though there is only an average of $8.1 \%$ of reduced average response time with only 10 samples, the variation of the result is huge, especially for the upper bound. In the worse case, the performance could be degraded by about $16 \%$ which is close to the performance without any clustering (see Figure 4), which is unacceptable.

\subsection{Adaption to Different Access Pattern}

The workload access pattern may not be the same over time. Our ASA-FTL is designed to track the data access hotness online so that it is able to adapt to any changes in the access pattern. This is a major difference comparing to existing data clustering methods that use static separation criteria. To evaluate the adaptivity of the ASA-FTL, we design

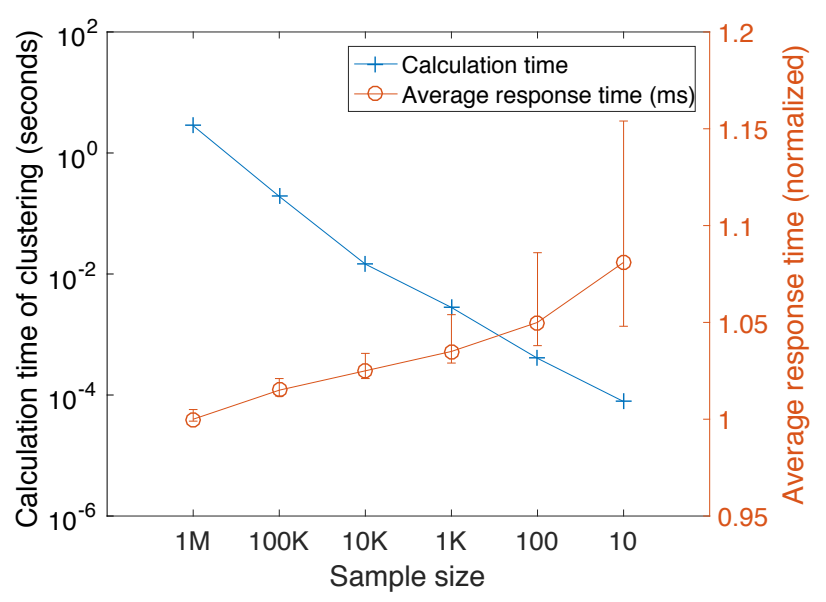

Figure 13: The effect of sample size for the calculation time of the cluster, the clustering error, and the average response time.

an experiment using the Synth9/10 trace described in Section 4.4. We slightly modify the Synth9/10 workload: we first write the first $10 \%$ address space with $90 \%$ requests and the rest with $10 \%$, and then after 30,000 requests we write the last $10 \%$ address space with $90 \%$ requests and the rest with $10 \%$ requests. Even though the general skewness is still the same, the data being hot and cold become different and failure to adapt to the change may result in a mixture of hot and cold data. Two sets of FTLs are used: one is DFTL with fixed separation criteria in which the first $10 \%$ address space is set as hot and rest set as warm; the other 


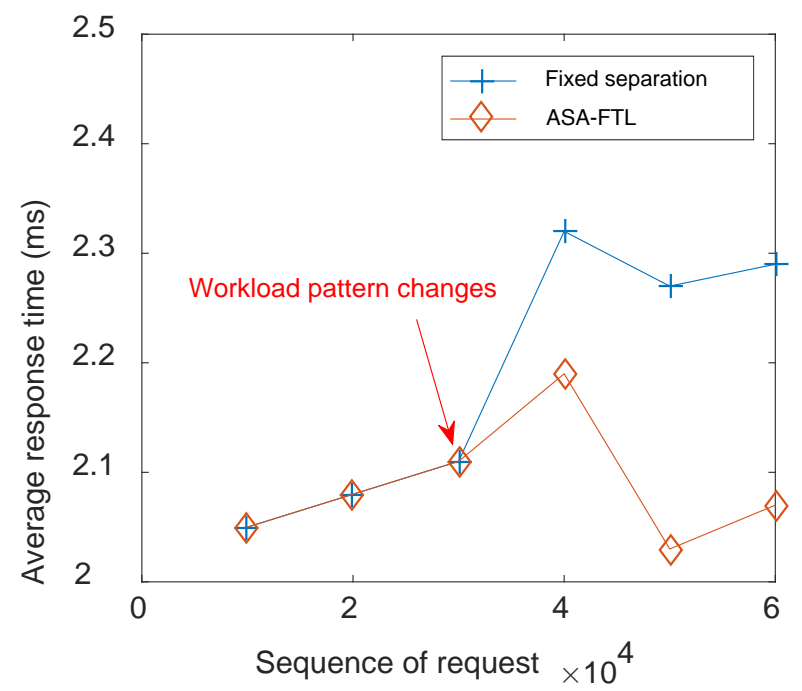

Figure 14: The adaptivity of ASA-FTL.

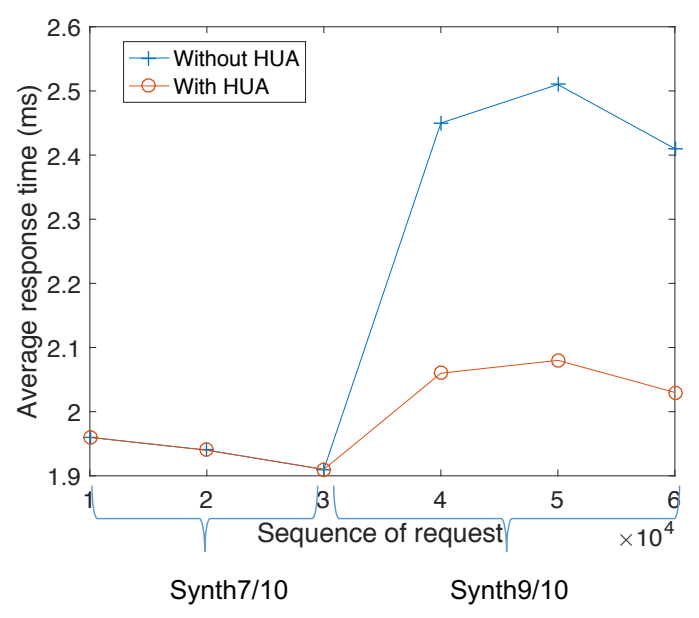

Figure 15: The adaption to workloads with different access skewness.

is the ASA-FTL (based on DFTL for the mapping) that is initialized with the same separation criteria. We record the average response time of every 10,000 requests.

In Figure 14, we find that both the ASA-FTL and the fixed separation perform the same for the first 30,000 requests. As the pattern changes at 30,000th request, the FTL with fixed separation starts to take more time to respond to requests. In contrast, the ASA-FTL performs significantly better than the fixed separation, even though it still sees some degree of degradation because it takes time to adapt to the new pattern. For the 40,000th to 50,000th requests, the ASAFTL has similar response time as before the pattern changes but the fixed separation FTL could not achieve the previous performance because it can not adapt to the change. The evaluation result matches our design goal that ASA-FTL is able to adapt to the changes in the access pattern and provide consistent improvement on the performance.
The above experiment assumes that the access skewness does not change, but it is usually not the case in real situations. We conduct another test to evaluate the HUA (Hotness Updating Algorithm)'s ability to adapt to different access skewness, i.e. to update the data separation criteria. In this test, we mix two synthetic workloads: synth7/10 and synth9/10. The simulated SSD is first warmed up with running the synth7/10 to give the ASA-FTL a chance to calculate the data separation criteria for it. The workload is switched from synth7/10 to synth9/10 at the 30000th write and two cases are considered then. In the first case "without HUA", the HUA is turned off while it is turned on in the second case "with HUA". It can be seen in Figure 15 that the HUA is able to outperform the case without HUA when the workload is switched to synth9/10. It indicates that HUA is able to adapt the data separation criteria to the new workload.

\section{RELATED WORK}

Numerous studies have focused on improving hot/cold data separation method for SSDs, improving garbage collection efficiency, and understanding the performance of SSDs. We discuss existing work along these three lines and compare the existing work with our work introduced in this paper.

\subsection{Hot/cold Data Separation}

There are several research studies on hot/cold data separation techniques in flash-based SSDs. Chiang et al 24 25. proposed an early online hot/cold data separation method that used write frequency to classify data as hot or cold. Other work through the years has also used write frequency or recency as the criteria defining data hotness 9, 26 29]. Shin 10 proposed a method by which any valid page found in the victim block selected by the garbage collector is classified as cold. This method is attractive in that it does not explicitly save track frequency or recency information while still providing good accuracy in classifying hot and cold data. Park et al 28, 29. proposed the use of Bloom filters for accurate and low-cost hot/cold data classification. This work noted the benefits of combining recency and frequency information for hot/cold data classification. The Bloom filter approach is also very efficient in terms of the memory required to implement the approach. For the FASTer 26] and ADAPT 27 projects, researchers recognized that a leastrecently used replacement policy is a natural hot/cold data classifier.

Our work differs from these existing work on classifying and separating hot/cold data in several fundamental ways:

1. The identification and separation of data happens whenever a page is written, whether due to a request in the workload or during garbage collection. The existing techniques only classify data during the cleaning phase. Although it is counter-intuitive, by considering data hotness more frequently, garbage collection overhead can be reduced.

2. Our approach uses IRR values for measuring hotness, rather than one or the other. This approach allows the identification of hotness without recording the access frequency of all pages, avoiding the considerable 
overhead caused by updating access frequency for all pages.

3. Our approach caches selected IRR values in RAM, and their values are only updated periodically to limit the overhead. This approach reduces ASA-FTL's onboard RAM requirement and avoids frequent updating of IRR values stored in the flash storage.

\subsection{Garbage Collection}

The garbage collection is considered the main cause of performance degradation of SSDs. Many researches tried to improve the garbage collection efficiency from different aspects. Chiang introduced the Cost/Age/Times calculation [24,30. for deciding the garbage collection victim, comparing to the traditional greedy and LRU policy for the cleaning victim selection. It determines the cleaning victim evaluating the Age of a data block and its estimated cost of cleaning. Thus the garbage collection efficiency is improved. Another method of improving the garbage collection efficiency is to insert a write buffer into the SSD. Data are buffered in cache and destaged to flash memory in groups. Hu et al. proposed PUD-LRU and GC-ARM 31,32, which improves the garbage collection efficiency by intelligently choosing the destaging policy based on access history. Their method significantly reduces the occurrence of cleaning and total overhead caused by garbage collection. This work is orthogonal to these methods in terms of reducing the garbage collection overhead and can be integrated into them to achieve better performance.

\subsection{Modeling and Analysis of SSD Performance}

Desnoyers et al 6, 33. conducted intensive research on modeling and analysis of the SSDs performance. Different workload patterns are considered for modeling and the optimal garbage collection policy are suggested. The non-uniform traffic is considered harmful for the performance. It suggests that hot/cold separation would optimize the performance of non-uniform traffic almost identical to that of the uniform traffic. The conclusion of their work is verified by our evaluations. The average response time of the simulations with ASA-FTL on Synth9/10, Synth7/10, and Synth5/10 are almost the same, which indicates that the performance of the SSD under non-uniform and uniform traffic are mostly the same, with the hot/cold separation integrated.

\section{CONCLUSION}

Solid-state drives (SSDs) have emerged as a highly compelling alternative to traditional hard disk drives in storage systems servicing data-intensive applications. However, the dominant SSD storage technology, NAND flash memory, is known to suffer performance degradation from the need of garbage collection due to its inherent limitations.

In this paper, we have proposed and evaluated a new data separation method, which is proven to be effective for controlling the cost of this garbage collection process. Our Adaptive Separation-Aware Flash Translation Layer, or ASAFTL, effectively classifies data as being hot, warm, or cold, and separates the data within the SSDs using a limited amount of on-board RAM and computation resources. The selective-caching and random-sampling methods are specifically designed in reducing the on-board RAM and processor usage, while still providing fine-grained, online data hotness identification and separation. The overhead of data clustering is reduced to an acceptable level with using only a small sample of the hotness information of all data pages in a flash memory. We have shown our approach to be especially effective for improving SSD performance when servicing workloads dominated by small write requests and with a relatively large degree of write access locality.

Our evaluation of ASA-FTL shows that it provides significant performance benefits if the workload features small writes and high locality, and performs at least as well as a state-of-the-art flash translation layer on real and synthetic benchmarks. The evaluation also indicates that the benefit of clustering is relatively insensitive to the sample size used, which makes using small sample size a reasonable choice. In addition, we show that ASA-FTL outperforms the fixedseparation methods when the access pattern of the workload changes, which indicates the better adaptivity of ASA-FTL with the help of the online tracking of pages' hotness information. Our long-term research agenda intends to advance the state-of-the-art storage techniques and promote better and highly efficient high-end/high-performance storage and computing systems for more productive data-intensive science.

\section{Acknowledgment}

This material is based upon work supported by the U.S. Department of Energy, Office of Science, Office of Advanced Scientific Computing Research. This research is supported by the National Science Foundation under grant CNS-1162488 and CNS-1338078.

\section{REFERENCES}

[1] S. M. Strande, P. Cicotti, R. S. Sinkovits, W. S. Young, R. Wagner, M. Tatineni, E. Hocks, A. Snavely, and M. Norman, "Gordon: Design, performance, and experiences deploying and supporting a data intensive supercomputer," in XSEDE '12, 2012, pp. 3:1-3:8.

[2] "Flash Technology in High-Performance Computing Accelerates Scientific Discovery," http://download.intel.com/newsroom/kits/xeon/phi/ pdfs/SSD_HPC_SDSC_CaseStudy.pdf

[3] T. Luo, R. Lee, M. Mesnier, F. Chen, and X. Zhang, "hstorage-db: heterogeneity-aware data management to exploit the full capability of hybrid storage systems," Proceedings of the VLDB Endowment, vol. 5, no. 10, pp. 1076-1087, 2012.

[4] F. Chen, D. A. Koufaty, and X. Zhang, "Hystor: Making the best use of solid state drives in high performance storage systems," in Proceedings of the International Conference on Supercomputing, ser. ICS '11. New York, NY, USA: ACM, 2011, pp. 22-32. [Online]. Available: http://doi.acm.org/10.1145/1995896.1995902

[5] W. Xie, J. Zhou, M. Reyes, J. Noble, and Y. Chen, "Two-mode Data Distribution Scheme for Heterogeneous Storage in Data Centers," in Big Data (Big Data), 2015 IEEE International Conference on, Oct 2015, pp. 327-332.

[6] P. Desnoyers, "Analytic Modeling of SSD Write Performance," in Proceedings of the 5th Annual 
International Systems and Storage Conference. ACM, 2012, p. 12.

[7] M. Rosenblum and J. K. Ousterhout, "The Design and Implementation of a Log-structured File System," ACM Transactions on Computer Systems (TOCS), vol. 10, no. 1, pp. 26-52, 1992.

[8] F. Chen, D. A. Koufaty, and X. Zhang, "Understanding Intrinsic Characteristics and System Implications of Flash Memory Based Solid State Drives," in Proceedings of the Eleventh International Joint Conference on Measurement and Modeling of Computer Systems, ser. SIGMETRICS '09. New York, NY, USA: ACM, 2009.

[9] C. Min, K. Kim, H. Cho, S. Lee, and Y. I. Eom, "SFS: Random Write Considered Harmful in Solid State Drives," in Proceedings of the 10th USENIX conference on File and Storage Technologies, FAST 2012, San Jose, CA, USA, February 14-17, 2012, 2012.

[10] I. Shin, "Hot/cold Clustering for Page Mapping in NAND Flash Memory," Consumer Electronics, IEEE Transactions on, vol. 57, no. 4, pp. 1728-1731, 2011.

[11] Q. Wei, B. Gong, S. Pathak, B. Veeravalli, L. Zeng, and K. Okada, "WAFTL: A Workload Adaptive Flash Translation Layer with Data Partition," in Mass Storage Systems and Technologies (MSST), 2011 IEEE 27th Symposium on. IEEE, 2011, pp. 1-12.

[12] A. Gupta, Y. Kim, and B. Urgaonkar, DFTL: A Flash Translation Layer Employing Demand-based Selective Caching of Page-level Address Mappings. ACM, 2009, vol. 44, no. 3 .

[13] S. Jiang and X. Zhang, "LIRS: an efficient low inter-reference recency set replacement policy to improve buffer cache performance," $A C M$ SIGMETRICS Performance Evaluation Review, vol. 30, no. 1, pp. 31-42, 2002.

[14] S.-W. Lee, D.-J. Park, T.-S. Chung, D.-H. Lee, S. Park, and H.-J. Song, "A Log Buffer-based Flash Translation Layer Using Fully-associative Sector Translation," ACM Transactions on Embedded Computing Systems (TECS), vol. 6, no. 3, p. 18, 2007.

[15] J. Kim, J. M. Kim, S. H. Noh, S. L. Min, and Y. Cho, "A Space-efficient Flash Translation Layer for Compact Flash Systems," Consumer Electronics, IEEE Transactions on, vol. 48, no. 2, pp. 366-375, 2002.

[16] "Financial1 I/O trace, U Mass Trace Repository ," http://traces.cs.umass.edu/index.php/

[17] J. A. Hartigan and M. A. Wong, "Algorithm AS 136: A K-Means Clustering Algorithm," Applied statistics, 1979.

[18] K. A. Nazeer and M. Sebastian, "Improving the accuracy and efficiency of the k-means clustering algorithm," in Proceedings of the World Congress on Engineering, vol. 1, 2009, pp. 1-3.

[19] R. Cheveresan, M. Ramsay, C. Feucht, and I. Sharapov, "Characteristics of Workloads Used in High Performance and Technical Computing," in Proceedings of the 21st Annual International Conference on Supercomputing, ser. ICS '07, 2007.

[20] Y. Kim, B. Tauras, A. Gupta, and B. Urgaonkar, "Flashsim: A Simulator for NAND Flash-based
Solid-state Drives," in Advances in System Simulation, 2009. SIMUL'09. First International Conference on. IEEE, 2009, pp. 125-131.

[21] J. S. Buch, J. Schindler, S. W. Schlosser, G. R. Ganger et al., "The DiskSim Simulation Environment Version 4.0 Reference Manual," 2008.

[22] N. Megiddo and D. S. Modha, "ARC: A Self-Tuning, Low Overhead Replacement Cache," in Proceedings of the 2Nd USENIX Conference on File and Storage Technologies, ser. FAST '03. Berkeley, CA, USA: USENIX Association, 2003.

[23] "IOTTA Repository, MSR/TPCC Cambridge Trace ," http://iotta.snia.org/tracetypes/3

[24] M.-L. Chiang, P. C. Lee, and R.-C. Chang, "Managing Flash Memory in Personal Communication Devices," in Consumer Electronics, 1997. ISCE'97., Proceedings of 1997 IEEE International Symposium on. IEEE, 1997, pp. 177-182.

[25] M.-L. Chiang, P. C. Lee, R.-C. Chang et al., "Using data clustering to improve cleaning performance for flash memory," SOFTWARE-PRACTICE \& EXPERIENCE, vol. 29, no. 3, pp. 267-290, 1999.

[26] S.-P. Lim, S.-W. Lee, and B. Moon, "FASTer FTL for Enterprise-class Flash Memory SSDs," in Storage Network Architecture and Parallel I/Os (SNAPI), 2010 International Workshop on. IEEE, 2010, pp. 3-12.

[27] C. Wang and W.-F. Wong, "ADAPT: Efficient Workload-sensitive Flash Management based on Adaptation, Prediction and Aggregation," in Mass Storage Systems and Technologies (MSST), 2012 IEEE 28th Symposium on. IEEE, 2012, pp. 1-12.

[28] J.-W. Hsieh, T.-W. Kuo, and L.-P. Chang, "Efficient identification of hot data for flash memory storage systems," ACM Transactions on Storage (TOS), vol. 2, no. 1, pp. 22-40, 2006.

[29] D. Park and D. H. Du, "Hot Data Identification for Flash-based Storage Systems using Multiple Bloom Filters," in Mass Storage Systems and Technologies (MSST), 2011 IEEE 27th Symposium on. IEEE, 2011, pp. 1-11.

[30] M.-L. Chiang and R.-C. Chang, "Cleaning Policies in Mobile Computers using Flash Memory," Journal of Systems and Software, vol. 48, no. 3, pp. 213-231, 1999.

[31] X.-Y. Hu, E. Eleftheriou, R. Haas, I. Iliadis, and R. Pletka, "Write Amplification Analysis in Flash-based Solid State Drives," in Proceedings of SYSTOR 2009: The Israeli Experimental Systems Conference. ACM, 2009, p. 10.

[32] J. Hu, H. Jiang, L. Tian, and L. Xu, "PUD-LRU: An Erase-efficient Write Buffer Management Algorithm for Flash Memory SSD," in Modeling, Analysis \& Simulation of Computer and Telecommunication Systems (MASCOTS), 2010 IEEE International Symposium on. IEEE, 2010, pp. 69-78.

[33] S. Boboila and P. Desnoyers, "Performance Models of Flash-based Solid-state Drives for Real Workloads," in Mass Storage Systems and Technologies (MSST), 2011 IEEE 27th Symposium on. IEEE, 2011, pp. 1-6. 